\title{
A Study on the Recommendation Model for Mobile Tourism Recommender System
}

\author{
Qi Wang, Yan Chen \\ Transportation Management College, Dalian Maritime University, Dalian, China \\ E-mail: 15940862235@163.com;1415958705@qq.com
}

\begin{abstract}
In recent years, with the continuous development of mobile communication technology, tourism recommender system based on the mobile terminal has become a hot spot in the domain of recommender systems. This paper introduces the concept of mobile tourism recommender system and the particularity of it, as well as the key techniques of mobile tourism recommender system. On the basis of two recommendation models including the general recommendation model and the recommendation model based on context in mobile environment, this paper presents a recommendation model for mobile tourism recommender system.
\end{abstract}

Keywords- recommendation model; mobile recommendation; tourism recommendation; mobile context

\section{INTRODUCTION}

With the rapid development of mobile communication technology and the rising popularity of smart mobile devices, smart mobile devices have gradually become the main platform of information acquiring. The user can access to information resources at any time and any place by searching network information through the mobile phone or tablet. Thus mobile network become the main channel for tourists to obtain information. Tourists can search tourism information online and buy tourism products or services. However, as the increase of mobile Internet services and information content, tourists overwhelmed with huge information.

It is difficult to make a choice in a lot of information. In addition, since the mobile device has some limitations, such as the interface display, terminal handling ability and the ability of interaction. So tourists are facing a serious "mobile information overload" problem [1]. In recent years, the mobile recommender system [2], taking advantages of the mobile network environment in the aspect of information recommendation and to overcome the adverse conditions, by capturing and forecasting potential mobile user preferences to filter irrelevant information, providing mobile users with personalized results, gradually become effective way of easing "mobile information overload" [3]. On the basis of existing studies, this paper will apply recommended mobile technology to tourism and study the recommendation model for mobile tourism recommender system.

\section{THE CONCEPT AND THE PARTICULARITY OF MOBILE TOURISM RECOMMENDER SYSTEM}

Mobile tourism recommender system using the mobile network environment to provide personalized results for mobile users, it is one of the main application of mobile recommender systems. Mobile travel recommendations not only include the recommendation of tourist attractions, but also involve the surrounding services (such as restaurants, hotels, etc.).

In the field of tourism, mobile recommender system is different from other recommended project. Collaborative filtering technology, and content-based filtering technology has been successfully applied to traditional fields, but it cannot simply be applied in the field of tourism [4]. Knowledge-based recommendation and hybrid recommendation is more suitable for mobile tourism recommender systems. This is mainly based on the following reasons. First, collaborative filtering method predicts whether a given user interest in a particular project depends on his past reviews, purchasing behavior and preferences of the community. But tourism experience is often limited and the cost is relatively high. So it is hard to obtain the user's feedback and purchasing history. Second, few people have the same travel experiences. We will find that the destination already visited is insufficient to predict the next one. Therefore, additional contextual information must be taken into account. Based on the complexity of tourism products and tourism decision-making, tourism recommendation generally requires the user to input some requirements. This requires an integrated method. Third, predicting the behavior of users can't just rely on historical rating data, but also consider the context information and user preferences [5]. For example, the type of travel (business or private), season, location, distance, personal interests and activities are important aspects of recommendation.

\section{KEY TECHNIQUES OF MOBILE TOURISM RECOMMENDER SYSTEM}

\section{A. Personalized Recommendation Technology}

1) Knowledge- based recommendation: Knowledgebased filtering technology uses the knowledge of the user and the product to produce recommendations, which uses a reasoning process to discover the product best suited to the needs of users [6]. This kind of technology without cold start problem, because it does not depend on the user's score. But this method needs a lot of domain knowledge and reasoning technology. Knowledge-based recommendation can be divided into three categories: constraint-based recommendation, case-based recommendation and knowledge-based reasoning recommendation. Tourism 
recommender system generally uses case-based recommendation technology.

2) Hybrid recommendation: Hybrid recommendation technology by integrating two or more recommendation technology can obtain better recommendation effect. It usually has four ways to achieve [7]. First, achieving content-based and collaborative filtering method respectively, and integrating the two prediction results. Second, adding content-based recommendation methods to collaborative filtering model. Third, adding collaborative iltering methods to content-based model. Forth, integrating content-based methods and collaborative filtering methods with a single rule. The most common practice is to combine the collaborative filtering technology with other certain kind of recommendation technology to overcome the cold start problem [8]. Hybrid recommendation methods make the full use of the advantages of various technologies to circumvent the lack of a certain technology to improve the recommended performance and accuracy.

\section{B. Recommendation Based on Mobile Context}

If the mobile recommender system does not consider the impact of context information, it cannot take advantage of the mobile recommendation effectively. Thus, the recommendation based on mobile context is widely concerned and researched. According to the binding mode of mobile context and mobile recommendation, the recommendation based on mobile context can be divided into mobile context filter and mobile context modeling. Mobile context filter using context to filter the set of recommended items, the items which are unrelated to the current context will be filtered out. Then, using conventional recommendation technologies (such as collaborative filtering) to produce recommendation results which meet the current context constraints and user's preferences [9]. Mobile context modeling integrates the context information into the recommendation procedure by designing recommendation algorithm, rather than filter the item set or recommendation results before recommending [10]. Integrating the context information into the recommendation model will make the recommendation model complicated, but this approach enables to combine the user's preferences with the context more closely and mine user's preferences more accurately. Mobile context filter converts "multidimensional recommendation" to "two-dimensional recommendation", so the integrity of the data cannot be guaranteed after dimensionality reduction and the recommendation effect will be affected [11].

\section{User Modeling Technology}

The user model of personalized recommender system is not a general description of the individual user, but a kind of description which is formal, algorithm oriented, and with a particular data structure [12]. User modeling is a process of generalizing a computable user model from user's interests and behaviors (such as browsing content, browsing behavior, background knowledge, etc.). Only on the basis of a high- quality user model, various goals that the personalized recommender system pursues can be achieved. User modeling technology is one of the main contents of the personalized information services. A complete user modeling process usually includes data collection, modeling and model updating.

\section{THE RECOMMENDATION MODEL FOR MOBILE TOURISM RECOMMENDER SYSTEM}

\section{A. General Recommendation Model}

Mobile tourism recommender system typically comprises three modules: data input module, personalized recommendation module and information output module. Data input module collects the user's search history and the behavior data. In addition, it understands the consumer's interest preferences and subdivides the user. Personalized recommendation module uses recommendation algorithms to process the data and get recommendation results. Information output module shows personalized recommendation results.

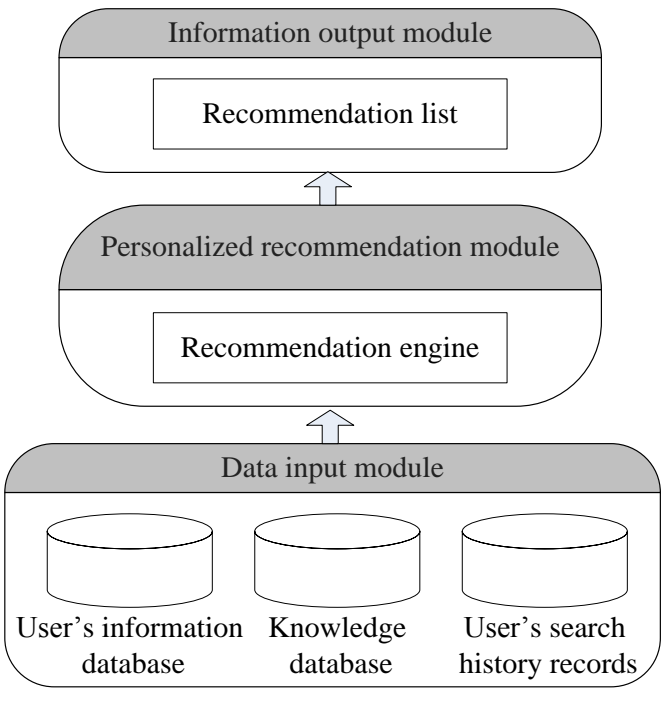

Figure 1. General recommendation model.

\section{B. The Recommendation Model Based on Context in Mobile Environment}

In the mobile Internet environment, the user's preferences will change with the change of the context, so the context information must be integrated into the recommendation model in order to obtain more accurate recommendation results. Context information can be obtained in the following ways: the sensor embedded in mobile devices (such as GPS module, accelerometer, timer, compass, gyroscope and camera), web services (such as weather reports or public transport information services), supporting facilities and other users. Examples of potential user's context parameters are location, distance, budget, time, season, idle time, transportation, weather conditions, moving history(such as scenic spots the user has visited), social environment. 
The portability of mobile devices makes more frequent interaction between users (such as mobile communication, mobile social network service), and makes the construction of mobile social networks easier and more reliable. Therefore, some mobile recommender systems also introduce the social networking service which not only can visit the city but also share their feelings after the tour. Users' activities (such as mobile mode, visited attractions, the pages viewed, etc.) are automatically recorded by the system. And the tourist-related contents (such as comments, attractions rating, photo, video, etc.) are also managed and shared at the same time.

Figure 2 shows the recommendation model based on context in mobile environment. In this model, the context information (including user context-aware information and social context-aware information) is introduced into the general model.

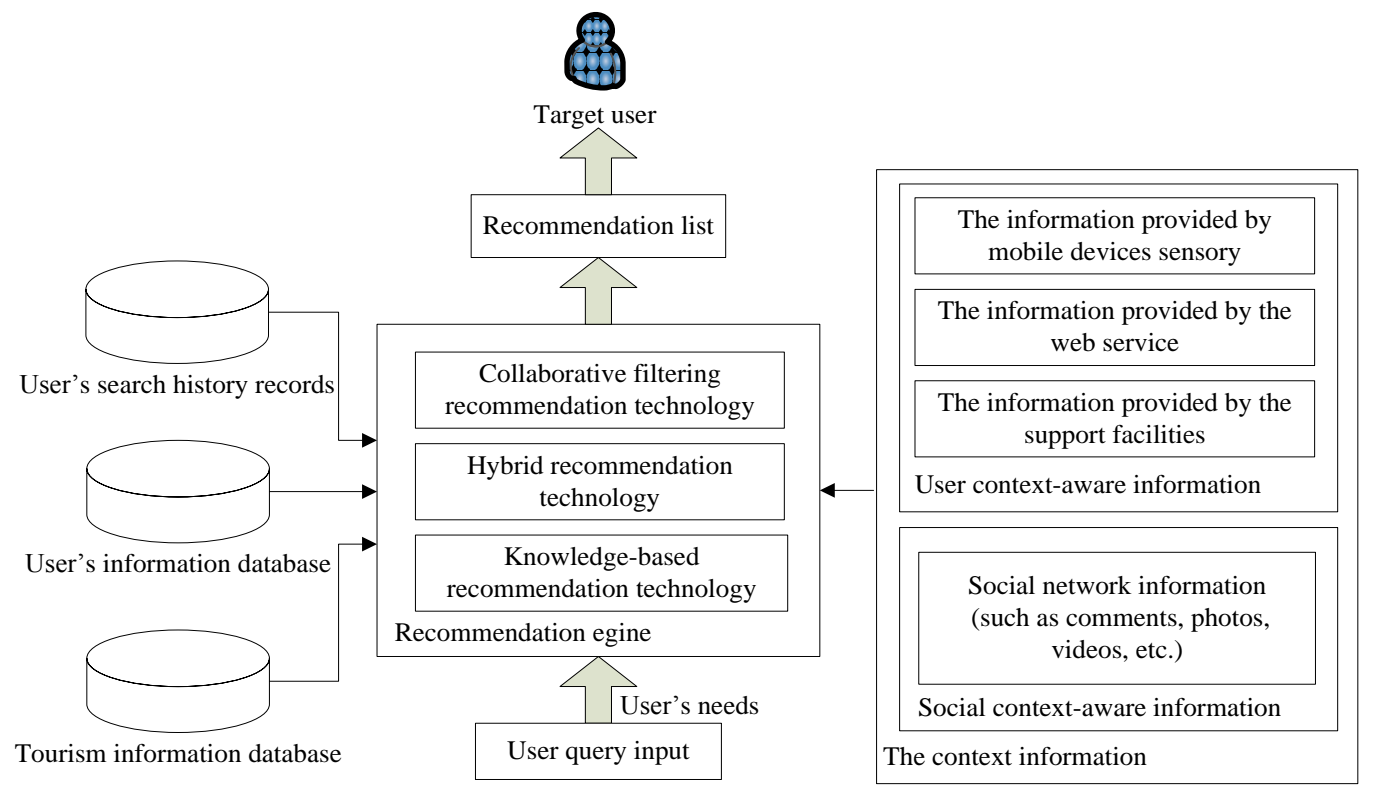

Figure 2. The recommendation model based on context in mobile environment. 


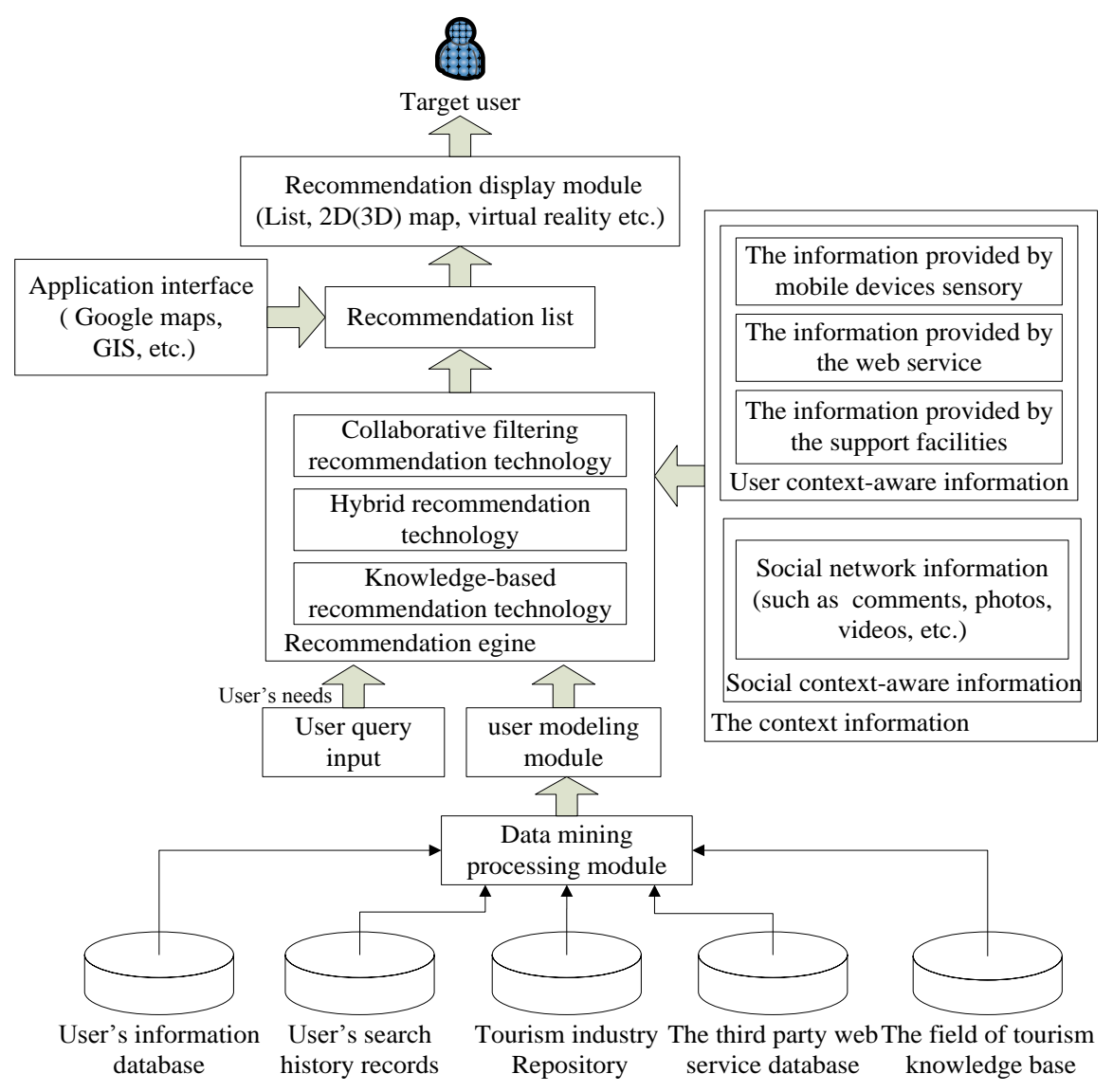

Figure 3. The extended recommendation model.

\section{Extended Recommendation Model}

In order to obtain more accurate recommendation results, extending the general recommendation model for mobile tourism recommender system, as shown in Figure 3. The model consists of five modules: the data mining processing module, the user modeling module, the context information module, the recommendation engine module, the recommendation display module. Through the collection, preparation and analysis of existing data and repository, the data mining processing module uses reasonable data mining algorithms to dig out the rules meet the needs of users. Based on data mining, the user modeling module is to create a user model and determine the user's profile information and interest preferences. Building the user model based on data mining modeling technology is able to dig out potential and useful information of the user, thus user's preferences can be accessed more accurately. The context information module as mentioned above, although it increases the complexity of the recommendation, but it improves the accuracy. According to all kinds of context information, the recommendation engine module produces personalized recommendation results and sorts the results. Application interfaces provide a variety of display forms and improve the visualization of recommendation results. The recommendation display module is to display the recommendation results in a list or $2 \mathrm{D}$ (3D) map in the mobile terminal by using application interfaces.

\section{CONCLUSION}

Although mobile tourism recommender system has become a hot spot in the domain of recommender systems, it still faces many challenges. The key contents of it are as follows: the intelligent human-computer interaction of mobile users, multi-modal identification, and comprehensive recommendation involves scenic tours, accommodation and catering. The recommendation model established in this paper will enhance the accuracy of recommendation by introducing the context information and user modeling based on data mining module, but there are still some deficiencies. The recommendation model for mobile tourism recommender system will be improved in the follow-up study, mainly includes the following aspects: building a specific context ontology model and a user model, optimizing recommendation algorithms, and the evaluation of recommendation results.

\section{ACKNOWLEDGMENT}

The authors are very grateful to the editors and reviewers for their valuable comments and suggestions. This work is partially supported by National Key Technology Research and Development Program of the Ministry of 
Science and Technology of China(No. 2014BAH24F04),National Natural Science Foundation of China (No. 71271034), the National Social Science Foundation of China (Grant No.15CGL031), the Fundamental Research Funds for the Central Universities (No. 3132016212), and Natural Science Foundation of Liaoning (No. 2014025015).

\section{REFERENCES}

[1] Wang LC, Meng XW, Zhang YJ."A cognitive psychology-based approach to user preferences elicitation for mobile network services," Acta Electronica Sinica,39(11): 2547-2553, 2011 (in Chinese with English abstract).

[2] Ricci F. "Mobile recommender systems." Int'1 Journal of Information Technology and Tourism,12(3):205-231, 2011

[3] Modsching M, Kramer R, Hagen KT, Gretzel U. Effectiveness of mobile recommender systems for tourist destinations: A user evaluation. In: Proc. of the Intelligent Data Acquisition and Advanced Computing Systems: Technology and Applications 2007. Washington: IEEE Computer Society. 663-668, 2007. [doi: 10.1109/IDAACS.2007.4488505].

[4] Jannach D, Gedikli F, Karakaya Z, et al. "Recommending hotels based on multi- dimensional customer ratings[A].//: Information and Communication Technologies in Tourism 2012[M]". Vienna: Springer. 320-331, 2012.
[5] Cao D, Nie W, Li R, et al. "Context-aware recommendation in mobile environments: An approach based on interest resonance[J]". Wuhan University Journal of Natural Sciences, 17(5): 400-406, 2012.

[6] Chen Jiemin, Tang Yong, Li Jianguo. "Survey of personalized recommendation algorithms [J]". South China Normal University: Natural Science Edition, 46 (5): 8-15. , 2014

[7] Song Leyi, Xiong Hui, Zhang Rong. "Towards the next generation of mobile recommender systems [J]". East China Normal University (Natural Science Edition), 3: 008. , 2013

[8] Liu Jianguo, Zhou Tao, Wang Binghong. "The progress of personalized recommender systems [J]". Progress in Natural Science, 19 (1): 1-15. , 2009

[9] Wang LC, Meng XW, Zhang YJ. "Context-Aware recommender systems. Ruanjian Xuebao/Journal of Software",23(1):1-20, 2012. (in Chinese with English abstract). Available: http://www.jos.org.cn/1000-9825/4100.htm [doi: 10.3724/SP.J.1001.2012.04100].

[10] Zhang Shijie. "A Study on context-aware collaborative filtering recommendation model in mobile environment [D]". Hangzhou Dianzi University, 2014.

[11] Xu Fengling, Meng Xiangwu, Wang Licai. "A collaborative filtering recommendation algorithm based on context similarity for mobile users [J]". Journal of Electronics and Information Technology, 33 (11): 2785-2789. , 2011

[12] Xu Qi. "Research on user modeling technology for personalized demands [J]". Science Technology and Engineering (11): 2655-2660, 2010 . 